DOI:

10.1038/nri2179

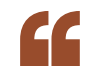

...'co-receptor

tuning' ensures

that $T$ cells

specific for self

antigens can

survive in the

periphery but

not be overtly

autoreactive.

antigens can survive in the periphery

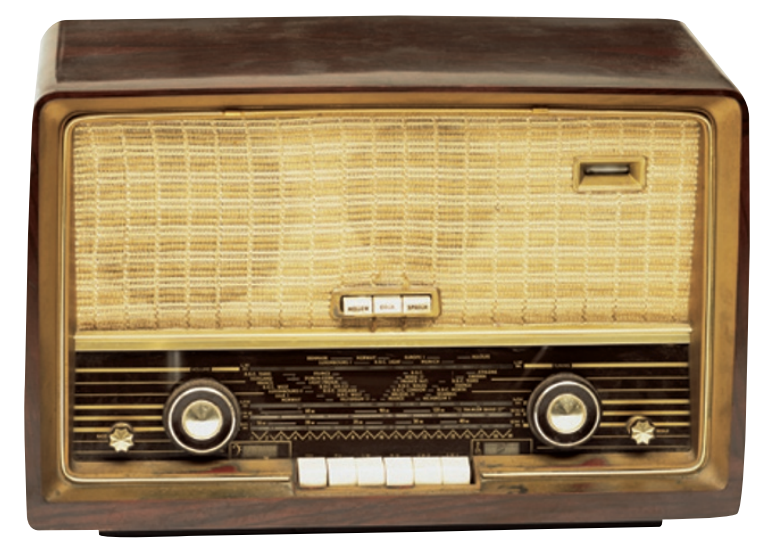
but not be overtly autoreactive.

The level of expression of the T-cell co-receptors CD4 and CD8 influences the intensity of signals through the T-cell receptor (TCR), such that high co-receptor expression increases signalling by relatively low-avidity TCRs whereas low co-receptor expression limits signalling by relatively high-avidity TCRs. But how is this regulated? Now, Alfred Singer and colleagues show that the level of CD8 expression is transcriptionally modified in mature $\mathrm{T}$ cells by signals from interleukin-7 (IL-7) and other cytokines of the common cytokine-receptor $\gamma$-chain $\left(\gamma_{c}\right)$ family. In turn, TCR signalling impairs the cell's responsiveness to cytokines and thereby decreases CD8 expression. This so-called 'co-receptor tuning' ensures that $\mathrm{T}$ cells specific for self
T CELLS

\section{Co-receptor tuning}

A simple culture assay revealed that CD8 expression may be dynamically regulated by cytokines, as CD8 expression decreased on mature $\mathrm{CD}^{+} \mathrm{T}$ cells if they were cultured overnight in medium alone, whereas it was retained if the culture medium was supplemented with IL-7 or other $\gamma_{c}$ cytokines. This cytokine-mediated maintenance of CD8 expression was shown to be at the level of upregulation of $C d 8 a$ transcription, which by itself is sufficient to modify the levels of CD8a-CD8b heterodimers on the cell surface.

Although IL-7 is constitutively produced by stromal cells, the authors considered the possibility that IL-7-mediated upregulation of CD8 expression might be influenced by signals through the TCR. To test this, the authors used the HY TCR-transgenic system because it is known that $\mathrm{T}$ cells in male mice expressing transgenic TCRs specific for the male antigen HY express low levels of CD8 and this is thought to prevent autoreactivity in these mice. Now, the authors show that CD8 expression by $\mathrm{T}$ cells from these male mice did not change after culturing overnight regardless of the presence or absence of IL-7, suggesting that they are refractory to IL-7 signals. However, CD8 expression could be upregulated by cytokines if the cells were cultured at low density, which is consistent with the idea that removal of the chronic HY antigen signal through the TCR restores responsiveness to cytokine signalling and subsequent CD8 expression.

Analysis of T cells transgenic for different TCRs showed that the level of CD8 expression inversely correlated with TCR signalling intensity (as measured by CD5 expression), implying that stronger TCR signals interrupt IL-7 receptor signalling more effectively and thereby suppress CD8 expression to a greater degree. Given that the level of CD8 expression in turn influences TCR signalling strength, these observations suggest a negative-feedback circuit that regulates $\mathrm{CD}^{+} \mathrm{T}$-cell reactivity in vivo - a mechanism the authors term co-receptor tuning.

Finally, the authors show that dysregulation of co-receptor tuning might contribute to autoimmunity, as the levels of CD5 and CD8 did not inversely correlate (both were highly expressed) in $\mathrm{T}$ cells from an autoimmune-prone mouse strain.

So, the interplay between IL-7 and TCR signals, both of which are needed for T-cell homeostasis, represents a new mechanism of peripheral tolerance of mature $\mathrm{CD} 8^{+}$ T cells whereby CD8 expression on individual $\mathrm{T}$ cells is adjusted to a level appropriate for their individual TCR, and explains how a self-reactive yet self-tolerant $\mathrm{T}$-cell repertoire is maintained.

Lucy Bird

ORIGINAL RESEARCH PAPER Park, J.-H. et al.

'Coreceptor tuning': cytokine signals transcriptionally tailor CD8 coreceptor expression to the self-specificity of the TCR. Nature Immunol. 8, 1049-1059 (2007) FURTHER READING Zúñiga-Pflücker, J. C. CD8 ${ }^{+} T$ cells are kept in tune by modulating IL-7 responsiveness. Nature Immunol. 8, 1027-1028 (2007) 